\title{
Determination of Fractured Rock's Representative Elementary Volume by a Numerical Simulation Method
}

\author{
Hatem Gasmi \\ College of Engineering, University of \\ Hail, Hail, Saudi Arabia and \\ University of Tunis El-Manar, \\ ENIT, Tunisia \\ gasmi_hatem@yahoo.fr
}

\author{
Essaieb Hamdi \\ University of Tunis El-Manar, \\ ENIT, Tunisia \\ essaieb@yahoo.fr
}

\author{
Mabrouk Touahmia \\ College of Engineering, \\ University of Hail \\ Hail, Saudi Arabia \\ touahmia@hotmail.com
}

\author{
Ahmed Torchani \\ College of Engineering, University of \\ Hail, Hail, Saudi Arabia and \\ University of Tunis, ENSIT, LISIER \\ Laboratory, Tunisia \\ tochahm@yahoo.fr
}

\author{
Attia Boudjemline \\ College of Engineering, University of Hail, \\ Hail Saudi Arabia \\ a.boudjemline@uoh.edu.sa
}

\begin{abstract}
The present study aims at developing a numerical program called DISSIM which can analyze the homogenization of rock massifs using a new subroutine which calculates Representative Elementary Volume (REV). The DISSIM methodology consists of two steps. The first step involves the modeling of the fractured network in order to provide a surface simulation that represents the real fracture of the examined front. The second step is to numerically model the wave propagation through the simulated fracture network while characterizing the attenuation of vibrations due to the effect of discontinuities. This part allows us to determine in particular the wave propagation velocity through the fractured mass, from which we can determine the homogenized Young's modulus. However, after extensive bibliographic research, it was realized that a third step appeared to be necessary. In fact, it is necessary to look for a representative elementary volume on which we apply the proposed homogenization method. Two types of the representative elementary volume are proposed in this article, the geometric REV and the mechanical REV. The presentation of these two types of REV and the DISSIM methodology are detailed in this paper. Then, this methodology was applied to the study of a real case. The present research provides a method allowing the calculation of both types of REV for fissured rocks. The case study yielded comparable results between the mechanical REV and the geometric REV, which is compatible with previous research studies.
\end{abstract}

Keywords-representative elementary volume; DISSIM methodology; homogenization; fractured rock; simulated fracture network

\section{INTRODUCTION}

The examination of a Representative Elementary Volume (REV) is the first step necessary for the homogenization of a studied rock mass. It is a matter of determining a sufficiently large medium with respect to the size of the heterogeneities. REV represents the size from which the homogenized magnitudes (mechanical, hydraulic, geometric, etc.) characterizing the heterogeneous medium become constant or their fluctuations become negligible. Different types of REV have been defined, like the hydraulic REV in [1-3]. Authors in [4] presented and analyzed the existence of a REV in a porous medium in which two fluids of different natures circulate. The mechanical REV has been studied in $[5,6]$ by different numerical homogenization methods. The geometric REV has been the subject of [7]. Other types such as the thermal REV [8] and the REV of the initial stresses, are also studied mainly for the massifs located at important depths. Three-dimensional (3D) fracture network is generated in [9] using field data and applied Particle Flow Code (PFC) to determine the effect of the representative elementary volume size. Other studies $[10,11]$ used numerical modeling based on Baecher's model [12] to determine REV. A combination of the procedures developed in $[13,14]$ and Monte Carlo simulation is implemented in [11] to create a large number of fracture network models to statistically represent the bedrock and analyze REV. It should be noted that knowing the size of REV makes it possible to analyze rock masses more precisely. Indeed, the results of the fracture networks simulation and numerical homogenization can only be applied to faithfully represent the real rock mass if the size of the simulation domain is greater than or equal to the REV's size. For this reason, the numerical method of homogenization DISSIM $[15,16]$ requires a new step for the REV. Therefore, two REV types of have been chosen to be computed: the geometrical REV and the mechanical REV, in order to have more accuracy in the choice of the simulation surface of the homogenized rock mass. 


\section{Methodology}

\section{A. Geometric REV Method}

The method used is to look for the smallest square whose center coincides with the center of the initial domain beyond which the value of the considered parameter does not change [7] (Figure 1).

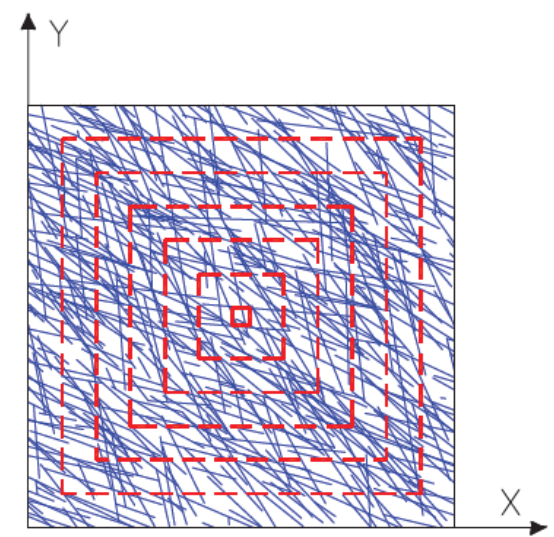

Fig. 1. Finding the size of the geometric REV: centered square of increasing size

The geometric REV's calculation is based on the average spacing value between fractures. It is defined as the size of the domain beyond which the mean spacing between fractures remains constant. As for the mean spacing, its calculation requires special analysis. Two spacings are determined in two different directions: Espvert in the vertical direction and Esphorz in the horizontal direction. The calculation steps are:

- According to the two considered directions, several parallel and equidistant sampling lines are considered.

- The number of fractures intercepting each line is calculated.

- The average spacing on each line is calculated by dividing the length of the sampling line by the number of fractures.

- The mean values of the two spacings, Espvert and Esphorz, of all sampling lines in the vertical direction and the horizontal direction respectively, are calculated using (1):

$$
\left\{\begin{array}{l}
\text { Espvert }=\sum \text { divert } / \text { pvert } \\
\text { Esphorz }=\sum \text { dihorz } / \text { phorz }
\end{array}\right.
$$

where, pvert and phorz are respectively the number of sampling lines in the vertical direction and the horizontal direction, and divert and dihorz are the vertical and horizontal spacings on each line.

\section{B. Search for the Mechanical REV}

Concerning the mechanical REV, and for each examined front, DISSIM can determine the equivalent Young's modulus by increasing the size of the simulated surface. Given the stochastic nature of the simulations from DISSIM program, 20 simulations can be performed. The average value obtained from the 20 simulations is taken as the Young's modulus value. The REV size is that of the simulation surface from which the Young's modulus becomes constant.

\section{NUMERICAL DEVELOPMENT OF DISSIM PROGRAM}

To apply the proposed REV research methodology, a subroutine has been developed which consists of different modules as follow:

- createHdr: In this module, successive domains are created from the initially simulated surface. After choosing the initial surface in the DISSIM module, the present subroutine creates centered squares of increasing surface starting with a domain of size $4 \mathrm{~m}$ until reaching the surface of the initial domain, with a step of $1 \mathrm{~m}$. The fracturing data for each created domain are saved in text files named Hdr_ $\mathrm{x}$ with $\mathrm{x}$ being the size of the domain in question.

- calculEsp: This module is used in the search of the geometric REV based on the calculation of the average spacing between fractures. For each size domain $\mathrm{x}$, the module starts by reading the existing fracture data in the square in question from file $\mathrm{Hdr} \mathrm{x}$. Then, parallel and equidistant sampling lines (equidistance $=1 \mathrm{~m}$ ) in both vertical and horizontal directions are created. For each sampling line, the average spacing between the fractures intercepting this line is calculated. The module will give as results on average vertical spacing and an average horizontal spacing for each domain. These results are recorded in a text file marked named ResEsp.

- calculEm: This module is used to find the mechanical REV based on the Young's modulus (Em) calculation. Similar to the preceding module, and for each size domain $\mathrm{x}$, the data of the network of discontinuities existing in the domain considered are read from the Hdr_x file. Subsequently, the wave propagation procedure is repeated by calling each time the modules mesh, Propag and Calculation developed in [16], in order to calculate the $E m$ for each created surface. Finally, the results generated by this calculation are saved in a text file called RES.

\section{REAL CASE STUDY}

In order to complete the numerical homogenization of the studied fronts, it is necessary to calculate their REV. A detailed example about the research of geometric REV and mechanical REV of the F13 front of the El Alto Quarry (Madrid, Spain) was presented in [16].

\section{A. Field Mapping Campaigns Using the Scanline Sampling Method}

The discontinuity survey method applied on the study front is the Scanline Sampling Method of the International Society for Rock Mechanics (ISRM). For each line, the parameters for any discontinuity through the scanline are systematically noted, namely, its location relative to a chosen reference (x), length, dip direction counted from the north axis, and dip angle 
measured from the horizontal plane. The field mapping campaigns of the F13 bench front are shown in Table I.

TABLE I. RESULTS OF THE FIELD MAPPING CAMPAIGNS USING THE SCANLINE SAMPLING METHOD (F13 BENCH FRONT)

\begin{tabular}{|c|c|c|c|c|c|c|c|}
\hline $\begin{array}{c}\text { Front } \\
\#\end{array}$ & $\begin{array}{c}\text { Line } \\
\#\end{array}$ & $\begin{array}{c}\text { Direction } \\
\left({ }^{\circ}\right)\end{array}$ & Discontinuity & $\begin{array}{c}X \\
(\mathrm{~cm})\end{array}$ & $\begin{array}{c}\text { Dip } \\
\text { direction } \\
\left({ }^{\circ}\right)\end{array}$ & $\begin{array}{l}\text { Dip } \\
\text { angle } \\
\left({ }^{\circ}\right)\end{array}$ & $\begin{array}{c}\text { Length } \\
\text { (cm) }\end{array}$ \\
\hline \multirow{44}{*}{ F13 } & \multirow{11}{*}{1} & \multirow{11}{*}{65} & 1 & 345 & 65 & 70 & 700 \\
\hline & & & 2 & 70 & 75 & 510 & 600 \\
\hline & & & 3 & 43 & 63 & 620 & 400 \\
\hline & & & 4 & 125 & 75 & 790 & 600 \\
\hline & & & 5 & 15 & 67 & 850 & 500 \\
\hline & & & 6 & 95 & 88 & 1110 & 300 \\
\hline & & & 7 & 101 & 90 & 1170 & 300 \\
\hline & & & 8 & 262 & 82 & 1250 & 100 \\
\hline & & & 9 & 235 & 76 & 1320 & 600 \\
\hline & & & 10 & 20 & 82 & 1410 & 500 \\
\hline & & & 11 & 215 & 82 & 1570 & 200 \\
\hline & \multirow{13}{*}{2} & \multirow{13}{*}{45} & 1 & 55 & 72 & 1650 & 300 \\
\hline & & & 2 & 255 & 85 & 1700 & 200 \\
\hline & & & 3 & 135 & 74 & 2020 & 600 \\
\hline & & & 4 & 35 & 85 & 2050 & 700 \\
\hline & & & 5 & 25 & 75 & 2130 & 500 \\
\hline & & & 6 & 50 & 85 & 2230 & 400 \\
\hline & & & 7 & 82 & 75 & 2400 & 300 \\
\hline & & & 8 & 74 & 64 & 2520 & 100 \\
\hline & & & 9 & 45 & 55 & 2630 & 500 \\
\hline & & & 10 & 15 & 67 & 3060 & 500 \\
\hline & & & 11 & 315 & 85 & 3160 & 500 \\
\hline & & & 12 & 137 & 85 & 20 & 300 \\
\hline & & & 13 & 132 & 84 & 30 & 700 \\
\hline & \multirow{9}{*}{3} & \multirow{9}{*}{60} & 1 & 5 & 79 & 320 & 600 \\
\hline & & & 2 & 35 & 73 & 330 & 500 \\
\hline & & & 3 & 98 & 69 & 360 & 200 \\
\hline & & & 4 & 326 & 80 & 460 & 400 \\
\hline & & & 5 & 326 & 80 & 490 & 400 \\
\hline & & & 6 & 107 & 75 & 510 & 500 \\
\hline & & & 7 & 82 & 65 & 540 & 200 \\
\hline & & & 8 & 82 & 65 & 560 & 200 \\
\hline & & & 9 & 82 & 65 & 600 & 200 \\
\hline & \multirow{11}{*}{4} & \multirow{11}{*}{285} & 1 & 32 & 74 & 180 & 300 \\
\hline & & & 2 & 69 & 54 & 520 & 500 \\
\hline & & & 3 & 55 & 52 & 610 & 300 \\
\hline & & & 4 & 55 & 52 & 650 & 300 \\
\hline & & & 5 & 332 & 85 & 1140 & 600 \\
\hline & & & 6 & 55 & 64 & 1510 & 500 \\
\hline & & & 7 & 333 & 88 & 1730 & 200 \\
\hline & & & 8 & 55 & 72 & 1650 & 300 \\
\hline & & & 9 & 255 & 85 & 1700 & 200 \\
\hline & & & 10 & 135 & 74 & 2020 & 600 \\
\hline & & & 15 & 35 & 85 & 2050 & 700 \\
\hline
\end{tabular}

\section{B. Geometric REV}

For domains of size ranging from $4 \mathrm{~m}$ to $20 \mathrm{~m}$ and with a step of $1 \mathrm{~m}$, the average spacing is calculated according to the domain size (Table II) for both sounding directions (vertical and horizontal). The variation in vertical and horizontal mean spacing is shown in Figure 2. It has been noticed that, for a size greater than $10 \mathrm{~m}$, the fluctuations of the value of the mean spacing become negligible in both directions as well as for the case of the average value which reaches a constant value of about $0.04 \mathrm{~m}$. From this result, the size of the geometrical REV was estimated at $10 \mathrm{~m}$. It has also been observed that for sizes greater than $17 \mathrm{~m}$, the average spacing starts to grow. This increase represents a perfectly explainable edge effect which schematizes a certain margin of error due to the absence of a few fractures, some of which exist inside the massif, but whose centers exist outside the domain. In this zone, the fracture density is lower which induces an increase in average spacing.

TABLE II. SPACING VALUES BY DOMAIN SIZE

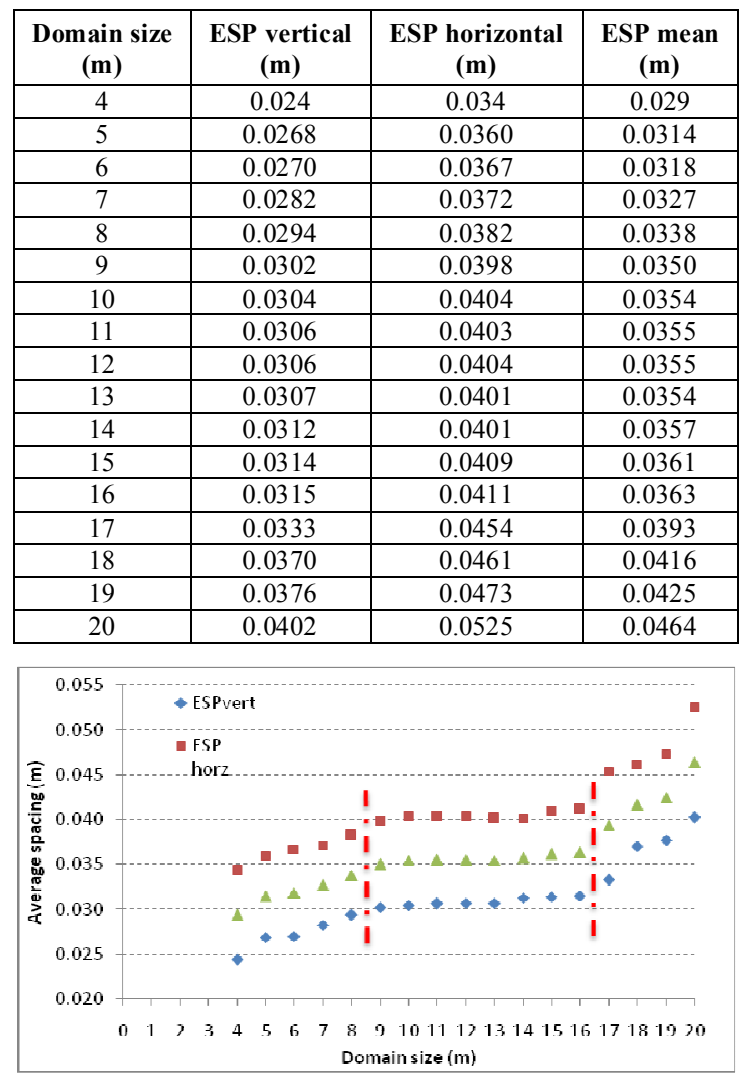

Fig. 2. Variation of vertical, horizontal and mean spacing of fractures as a function of domain size

\section{Mechanical REV}

The parameter chosen in the search for the mechanical REV is the Young's modulus of the rock mass. In the case of the F13 front, the mean value of the Young's modulus is calculated for each domain whose size varies between $4 \mathrm{~m}$ and $20 \mathrm{~m}$ with a pitch of $1 \mathrm{~m}$. It should be noted that the maximum domain size can be increased if the REV is not reached within the proposed range. Table III presents the results of the calculation of the mechanical REV for the F13 front. It includes the value of the average Young's modulus for each domain. This average value is calculated on the basis of 20 simulations carried out for each domain. The variation of the Young's modulus as a function of the domain size is shown in Figure 3. Similar to the spacing calculation, it has been observed that for a size greater than $11 \mathrm{~m}$, the fluctuations of Young's modulus become negligible. This parameter reaches a constant value of approximately 4.1GPa. Based on this result, the size of the mechanical REV has been estimated at $11 \mathrm{~m}$. According to the literature, as long 
as the mechanical properties of the rock and the joints do not vary in the field, the geometrical REV and the mechanical REV are equivalent.

TABLE III. AVERAGE VALUES OF YOUNG'S MODULUS

\begin{tabular}{|c|c|}
\hline Domain size(m) & Em (GPa) \\
\hline 4 & 1.05 \\
\hline 5 & 1.46 \\
\hline 6 & 1.89 \\
\hline 7 & 2.35 \\
\hline 8 & 2.78 \\
\hline 9 & 3.20 \\
\hline 10 & 3.67 \\
\hline 11 & 4.14 \\
\hline 12 & 4.10 \\
\hline 13 & 4.11 \\
\hline 14 & 4.16 \\
\hline 15 & 4.14 \\
\hline 16 & 4.12 \\
\hline 17 & 4.08 \\
\hline 18 & 4.50 \\
\hline 19 & 4.58 \\
\hline 20 & 4.70 \\
\hline
\end{tabular}

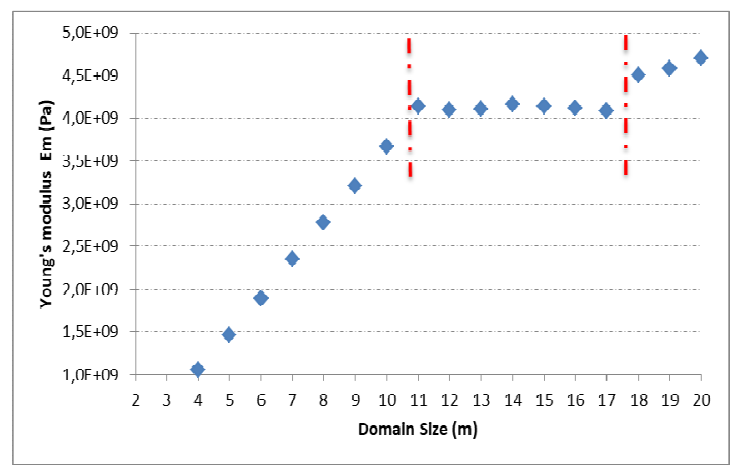

Fig. 3. Value of the equivalent Young's modulus according to domain size

\section{CONCLUSION}

A numerical program called DISSIM has been developed. This tool, programmed under MATLAB environment, uses the ISRM methodology to develop useful information on the structure of a rock mass. In general, the following results were obtained:

- In order to push the analysis to the response of the rock mass with respect to the blast-induced propagation of variation, a subroutine was added to DISSIM to calculate the representative elemental volume of the studied mass. It consists of two modules: the first one to search for the mechanical REV and the second for the geometric REV.

- To validate the numerical tool, the working methodology has been applied to real mass cases whose fracturing is quite complicated. It has been noted that the estimated geometric REV and mechanical REV are of comparable size. This is consistent with the findings of $[7,17]$.

- The size of the REV is estimated at $11 \mathrm{~m}$ for the F13 front. This size is retained for the remaining of the homogenization calculation for this front.

\section{ACKNOWLEDGMENT}

The present work was undertaken within the research project (No. 160717), funded by the Deanship of Scientific Research, at the University of Hail.

\section{REFERENCES}

[1] J. C. S. Long, J. S. Remer, C. R. Wilson, P. A. Withespoon, "Porous media equivalents for networks of discontinuous fractures", Water Ressources Research, Vol. 18, No. 3, pp. 645-658, 1982

[2] A. Pouya, A. Courtois, "Definition de la permeabilite equivalente des massifs fractures par des methodes d'homogeneisation", Comptes Rendus Geoscience, Vol. 334, No. 13, pp. 975-979, 2002 (in French)

[3] A. Pouya, "Tenseurs de permeabilite equivalente d'un domaine heterogene fini", Comptes Rendus Geoscience,Vol. 337, No. 6, pp. 581588, 2005 (in French)

[4] L. Zhang, L. Xia Q. C. Yu, "Determining the REV for Fracture Rock Mass Based on Seepage Theory", Geofluids, Vol. 2017, Article ID: 4129240, 2017

[5] K. B. Min, L. Jing, "Numerical determination of the equivalent elastic compliance tensor for fractured rock masses using the distinct element method", International Journal of Rock Mechanics and Mining Sciences, Vol. 40, No. 6, pp. 795-816, 2003

[6] L. Xia, Y. Zheng, Q. Yu, "Estimation of the REV size for blockiness of fractured rock masses", Computers and Geotechnics, Vol. 76, pp. 83-92, 2016

[7] M. Chalhoub, A. Pouya, "A geometrical approach to estimate the mechanical REV of a fractured rock mass", 1st Euro Mediterranean Symposium on Advances on Geomaterials and Structures, Hammamet, Tunisia, May 3-5, 2006

[8] K. B. Min, J. Lee, O. Stephansson, "Implications of thermally-induced fracture slip and permeability change on the long-term performance of a deep geological repository", International Journal of Rock Mechanics and Mining Sciences, Vol. 61, pp. 275-288, 2013

[9] M. Pierce, P. Cundall, D. Potyondy, D. M. Ivars, "A synthetic rock mass model for jointed rock", in: Rock Mechanics: Meeting Society's Challenges and Demands, Vol. 1, pp. 341-349, Taylor \& Francis Group, 2007

[10] W. S. Dershowitz, H. H. Einstein, "Characterizing rock joint geometry with joint system models", Rock Mechanics and Rock Engineering, Vol. 21, pp. 21-51, 1988

[11] W. Zhang, J. P. Chen, C. Liu, R. Huang, M. Li, Y. Zhang, "Determination of Geometrical and Structural Representative Volume Elements at the Baihetan Dam Site", Rock Mechanics and Rock Engineering, Vol. 45, pp. 409-419, 2012

[12] G. B. Baecher, N. A. Lanney, H. H. Einstein, "Statistical description of rock properties and sampling", 18th U.S. Symposium on Rock Mechanics, Colorado, USA, June 22-24, 1977

[13] J. Chen, "3-D network numerical modeling technique for random discontinuities of rock mass", Chinese Journal of Geotechnical Engineering, Vol. 23, No. 4, pp. 397-402, 2001

[14] J. Chen, B. F. Shi, Q. Wang, "Study on the dominant orientations of random fractures of fractured rock masses", Chinese Journal of Rock Mechanics and Engineering, Vol. 24, No. 2, pp. 241-245, 2005

[15] H. Gasmi, S. Yahyaoui, E. Hamdi, "A new tool for homogenization of jointed rock masses using wave propagation analysis", 10th International Symposium on Rock Fragmentation by Blasting, New Delhi, India, November 26-29, 2012

[16] H. Gasmi, E. Hamdi, N. B. Romdhane, "A Numerical homogenization of jointed rock masses using wave propagation simulation", Rock Mechanics and Rock Engineering, Vol. 47, No. 4, pp. 1393-1409, 2014

[17] K. Esmaieli, J. Hadjigeorgiou, M. Grenon, "Estimating geometrical and mechanical REV based on synthetic rock mass models at Brunswick Mine", International Journal of Rock Mechanics and Mining Sciences, Vol. 47, No. 6, pp. 915-926, 2010 